\title{
How vesicles put on their coat
}

Single-molecule imaging helps researchers to begin a biography for clathrincoated vesicles.

Many membrane-bound proteins hitch a ride in clathrin-coated vesicles to other cellular compartments, and scientists have spent decades studying the steps by which these vesicles form. The early stages involve the formation of 'coated pits' via the accumulation of molecules of clathrin protein linked to AP2 adaptor protein complexes, but the actual initiating events have remained a matter of debate.

By examining coated-pit assembly at the single-molecule scale, a team led by Harvard University cell biologist Tomas Kirchhausen and including postdoctoral fellows Emanuele Cocucci and François Aguet has now put together a storyline for these early events. Kirchhausen and others have previously used confocal microscopy-based techniques to study clathrin-mediated endocytosis in live cells, but these approaches could not deliver single-molecule resolution. This time, his team employed total-internal-reflection fluorescence, which enables high-resolution imaging of events occurring near the surface of a biological sample.

Clathrin forms three-legged structures known as triskelions: three heavy-chain polypeptides form the legs, and each heavy chain is in turn attached to a smaller light-chain polypeptide. The researchers expressed an EGFP-labeled light-chain construct in cultured monkey kidney cells to generate labeled triskelions. Because coated pits are relatively fixed in place by connections to the plasma membrane, the team could perform imaging at timescales suitable for single-molecule visualization. "It is hard to visualize these molecules if they are free because they're moving too fast and the fluorescence signal appears as just a blur," says Kirchhausen. "But because these triskelions are anchored, we could simply take a coated pit that is forming and then walk things back in time and try to pick up the beginning of the pit."

His team identified 537 pit formation events after 1 minute of imaging in five different cells. They consistently observed that each of these events began with two stepwise increases in fluorescence. Because EGFP-labeled clathrin was expressed alongside endogenous unlabeled clathrin, a given triskelion might have borne anywhere from zero to three EGFP labels. As such, it was necessary to perform in-depth modeling and analysis to determine exactly how many triskelions were being incorporated at each step. Kirchhausen and colleagues ran a variety of simulations, attempting to predict the molecular events that would be most likely to yield the observed experimental outcomes.

They determined that in the great majority of cases, the first two steps each entail incorporation of a single triskelion. Similar analysis with labeled AP-2 indicated that this molecule tends to get incorporated in pairs, allowing Kirchhausen and colleagues to devise a model for the first several seconds of pit formation. "You would not expect to have clathrin bound to its adaptor in solution because it's a very weak interaction," he says. "So the logic is, you have adaptors that arrive at the membrane and, if you have two adaptors that get clamped by one triskelion, that's going to be relatively stable; then a second clathrin can come and interact with the first one." As triskelions bind to each other at multiple sites, the stability of the growing clathrin coat rapidly increases. Importantly, this process appears to be highly stochastic and dependent on the timing of clathrin-AP2 interactions rather than actively induced by events at the membrane, as some researchers have previously proposed.

Kirchhausen now intends to move forward in the process and examine how coated pits successfully bud off as mature vesicles. However, he also sees this approach as a generalizable strategy for characterizing other membraneassociated events. "In my mind, this could be used to analyze many other processes in the cell," he says, "whether it's assembly of a signaling platform, formation of a nuclear pore or viral budding."

Michael Eisenstein

\section{RESEARCH PAPERS}

Cocucci, E., et al. The first five seconds in the life of a clathrin-coated pit. Cell 150, 495-507 (2012). 